\title{
VARIACIÓN DE LA ESTABILIDAD Y ALTURA DE LA CAPA DE MEZCLA EN LA CIUDAD DE PINAR DEL RÍO: SU RELACIÓN CON CONDICIONES SINÓPTICAS
}

\author{
DAGOBERTO RODRÍGUEZ VALDÉS ${ }^{1}$, ARELY QUINTERO SILVERIO ${ }^{2}$, YOSDANY GONZÁLEZ \\ JAIME $^{3}$, OSVALDO CUESTA SANTOS ${ }^{3}$, ALMARA SÁNCHEZ DÍAZ ${ }^{4}$
}

${ }^{1}$ Universidad de Pinar del Río, Facultad de Ciencias Técnicas, Departamento de Física, Pinar del Río, PR, Cuba

${ }^{2}$ Universidad de Pinar del Río, Facultad de Ciencias Técnicas, Departamento de Matemáticas, Pinar del Río, PR, Cuba

${ }^{3}$ Instituto de Meteorología de Cuba, Centro de Contaminación y Química Atmosférica, La Habana, LH, Cuba

${ }^{4}$ Instituto de Meteorología de Cuba, Centro Meteorológico Provincial de Pinar del Río, Pinar del Río, PR, Cuba

dagoberto@vrect.upr.edu.cu, arelys@mat.upr.edu.cu,yosdany.gonzalez@insmet.cu, almara@pri.insmet.cu

Ricibido Febrero de 2014 - Aceptado Octubre de 2014

\begin{abstract}
RESUMEN
El estudio se realizó en la ciudad de Pinar del Río en Cuba durante el período 2006-2010, donde se analizó el comportamiento de la estabilidad atmosférica y altura de la capa de mezcla. Para obtener la categoría de estabilidad atmosférica se utilizó la clasificación de acuerdo a la longitud de MoninObukhov. La metodología para calcular la altura la capa de mezcla, estuvo basada en el procedimiento establecido en el preprocesador meteorológico AERMET, con algunas modificaciones por no disponer sondeos de aire superior. Además se caracterizaron los comportamientos de la estabilidad atmosférica y altura de la capa de mezcla en los diversos Tipos de Situaciones Sinópticas (TSS). Finalmente se muestran la variación horaria y mensual de la estabilidad atmosférica y de la altura de la capa de mezcla, así como la variación mensual a las 7,13,16 y 17 horas. Se pudo determinar que las mejores condiciones para la dispersión y el mezclamiento de los contaminantes en la atmósfera ocurren en los meses de verano y en el horario diurno, cuyo horario más favorable se presenta a las 17 horas.
\end{abstract}

Palabras Claves: estabilidad atmosférica; capa de mezcla; tipos de situaciones sinópticas.

\begin{abstract}
STABILITY AND CHANGE IN MIXING HEIGHT IN THE CITY OF PINAR DEL RIO: IT'S RELATIONSHIP WITH SYNOPTIC CONDITIONS

The study was carried out in the city of Pinar del Rio in Cuba during the period 2006-2010, where the behavior of atmospheric stability and the mixing height was analyzed. For obtaining the category of atmospheric stability the classification according to the Monin -Obukhov length was used. The methodology for calculating the mixing height was based on the procedure in the meteorological preprocessor AERMET, with some modifications for lack of upper air soundings. Also the behavior of the atmospheric stability and the mixing height in the various types of synoptic situations (TSS) were characterized. Finally the hourly and monthly variations of atmospheric stability and height of the mixing layer, as well as the 7, 13, 16 and 17 hours monthly variations are shown. It was determined that the best conditions for dispersion and mixing of pollutants in the atmosphere occur in the summer months and in the daytime, 5PM as the most favorable schedule time.
\end{abstract}

Keywords: atmospheric stability; mixing height; types of synoptic situations.

\section{INTRODUCCIÓN}

Los principales avances teóricos en el conocimiento de la turbulencia de la atmósfera se han desarrollado simultáneamente a lo largo del siglo xx. Así, la teoría estadística de la turbulencia y los problemas relativos a la difusión, según se muestra en (Garratt, 1992), se deben en gran medida a los trabajos realizados por Taylor. En la década de los cincuentas y principio de los sesentas del siglo XX, se realizaron grandes avances en el conocimiento de los procesos turbulentos bajo condiciones de flotabilidad y 
se desarrollaron las relaciones de flujo-gradiente. De este modo, a partir de los trabajos desarrollados por (Monin y Obukhov, 1954), nace la teoría de similitud acerca de la capa superficial.

En 1991, la Sociedad Meteorológica Americana (AMS, siglas en inglés) y la Agencia de Protección Ambiental de los EE.UU. (EPA, siglas en inglés), iniciaron una colaboración formal con el objetivo de introducir a los conceptos conocidos de capa límite y altura de la capa de mezcla, nuevos conceptos como la Capa Límite Planetaria (PBL, siglas en inglés) en los modelos de dispersión de reglamentación. Las principales novedades en la comprensión de la PBL se iniciaron en la década de 1970 (Venkatram y Wyngaard, 1988). Para la Capa Límite Convectiva (CBL, siglas en inglés), Deardorff (1972) realizó estudios que revelaron la estructura vertical del CBL y las importantes escalas de la turbulencia.

Las principales ideas sobre la dispersión, seguidas a partir de experimentos de laboratorio, simulaciones numéricas y observaciones de campo, se obtuvieron en los trabajos de (Izumi et al, 1971), (Readings et al, 1974), (Lamb, 1982) y (Weil, 1988). Para la Capa Límite Estable (SBL, siglas en inglés), los avances se produjeron más lentamente. Sin embargo, un sólido marco teórico/experimental para la dispersión y la capa superficial con enfoques para fuentes elevadas emergieron a mediados de 1980 (Venkatram, 1988). Durante mediados de 1980, los investigadores: (Weil, 1985), (Pasquill y Smith, 1983), (Nieuwstadt y van Dop, 1982), (Venkatram y Wyngaard, 1988) empezaron a aplicar esta información a modelos de dispersión simples. En una revisión de los modelos regulatorios existentes o propuestas desarrolladas antes de 1984 (Smith, 1984), (Hayes y Moore, 1986) informaron que las técnicas de años anteriores, produjeron predicciones que no estaban de acuerdo con las observaciones. La necesidad de una reforma integral de la normativa de los modelos básicos de la EPA se reconoció claramente. El nuevo modelo desarrollado por AERMIC (AMS/ EPA Regulatory Model Improvement Committee) está dirigido a la dispersión de corto plazo desde fuentes estacionarías, el mismo escenario manejado por la EPA en ISC3 (Complejo de Fuente Industrial de Corto Plazo), (EPA, 1995).

En Cuba se han realizado importantes estudios sobre modelación de la calidad del aire, aplicando los modelos de última generación para fuentes estacionarias como el ISC3 y más reciente el AERMOD (AMS/EPA Regulatory MODel), resultando primordial conocer el comportamiento de la altura de la capa de mezcla (APCM) y de la estabilidad atmosférica (EA). Existiendo en Cuba trabajos dirigidos a conocer este comportamiento que datan desde la década de los setentas del siglo XX: (Álvarez, 1976) y (López, 1978) y más reciente (Turtós et al, 2003, 2009), (Rodríguez, 2007), (Rodríguez et al, 2008, 2012a y 2012b), aplicando básicamente los métodos de (Turner, 1964) para obtener la estabilidad atmosférica y de
(Pasquill, 1961) para conocer la altura de la capa de mezcla. Pero resultan muy escasos los estudios relacionados con la influencia de las condiciones sinópticas sobre el comportamiento de la estabilidad y altura de la capa de mezcla en Cuba y solo se muestra el reciente resultado de (Alonso, 2013).

En la ciudad de Pinar del Río, provincia homónima de Cuba, la mayoría de las fuentes estacionarias, presentan tecnologías atrasadas debido al tiempo de explotación, incidiendo esto negativamente en las emisiones de contaminantes. Agravando la situación, la ubicación neurálgica de algunas, específicamente las que están en pleno sector urbano, conllevando al deterioro de la calidad del aire, afectando a la población y al medio ambiente de forma general.

En Cuba al igual que en muchos países en vías de desarrollo, no se realizan sondeos de la atmósfera superior, por no disponer del equipamiento necesario. Por lo que constituye una alternativa importante "obtener el comportamiento de la estabilidad atmosférica y de la altura de la capa de mezcla y su relación con los sistemas sinópticos en la ciudad de Pinar del Río, aplicando métodos teóricos", constituyendo este el objetivo del trabajo.

\section{MATERIALES Y MÉTODOS}

\subsection{Clasificación de la estabilidad atmosférica}

La estabilidad atmosférica se obtuvo para condiciones estables y convectivas, mediante la clasificación de la Longitud de Monin-Obukhov (L) (Gryning et al, 2007), ver Tabla 1.

\subsection{Obtención de la altura de la capa de mezcla atmosférica}

El cálculo de la altura de la capa de mezcla atmosférica se realizó de la siguiente forma:

Tabla 1 - Clasificación de los intervalos de Longitud de MoninObukhov.

\begin{tabular}{|c|l|c|}
\hline ID & \multicolumn{1}{|c|}{ Clasificación } & Intervalo de L \\
\hline 1 & Muy estable (ME) & $10 \leq L<50$ \\
\hline 2 & Estable (E) & $50 \leq L<200$ \\
\hline 3 & Ligeramente estable (LE) & $200 \leq L<500$ \\
\hline 4 & Neutra (N) & $|L| \geq 50$ \\
\hline 5 & Ligeramente inestable (LI) & $-500 \leq L<-200$ \\
\hline 6 & Inestable (I) & $-200 \leq L<-100$ \\
\hline 7 & Muy inestable (MI) & $-100 \leq L<-50$ \\
\hline
\end{tabular}


Durante condiciones estables, cuando $L>0$, la altura de la capa de mezcla atmosférica $(Z i)$ es igual a la altura de la capa de mezcla mecánica (Zim), el mismo método seguido en EPA (2004a).

Durante condiciones convectivas, cuando $L<0$, la altura de la capa de mezcla atmosférica $(Z i)$ es el máximo entre la altura convectiva (Zic) y mecánica (Zim). Se realizó mediante el procedimiento desarrollado en el documento (EPA, 2004a) y el trabajo de (Turtós et al., 2009) y se muestra a continuación.

\subsection{Altura de la capa de mezcla atmosférica durante condiciones convectivas}

Para calcular la altura de la capa de mezcla convectiva (Zic), se utiliza la formulación de (Batchvarova y Gryning, 1991).

$$
\frac{\partial Z i c}{\partial t}=(1+2 A) \frac{H}{\rho C_{p} \gamma Z i c}+2 B \frac{u_{*}^{3} T_{r e f}}{\gamma g Z i c^{2}}
$$

Donde: $A$ y $B$, constantes adimensionales $(A=0.2$ y $B=5) ; H$, el flujo de calor; $\rho$, densidad del aire; $C_{P}$, calor específico a presión constante; $g$, aceleración de la gravedad; $u_{*}$, velocidad de fricción; $T_{r e f}$, temperatura de referencia (de superficie); $\gamma$ ,gradiente de temperatura potencial sobre la capa de mezcla, propuesta por (Gill, 1982):

$$
\gamma=N u^{2} T_{\text {ref }} / g
$$

Donde: $N u$ es la frcuencia de Brunt-Väisälä, cuyo valor sugerido por (Thomson, 2000) es de $0.013 \mathrm{~s}^{-1}$.

La Ecuación 1 fue resuelta numéricamente por (Turtós et al., 2009), considerando que para pequeños intervalos de tiempo, la variación de Zic con respecto al tiempo, puede ser aproximada en diferencias finitas:

$$
\partial Z i c / \partial t=\Delta Z i c / \Delta t
$$

Considerando $\Delta t=360 \mathrm{~s}$. La (Zic) se puede calcular a partir del valor en el instante precedente, a partir de (Turtós et al., 2009) como:

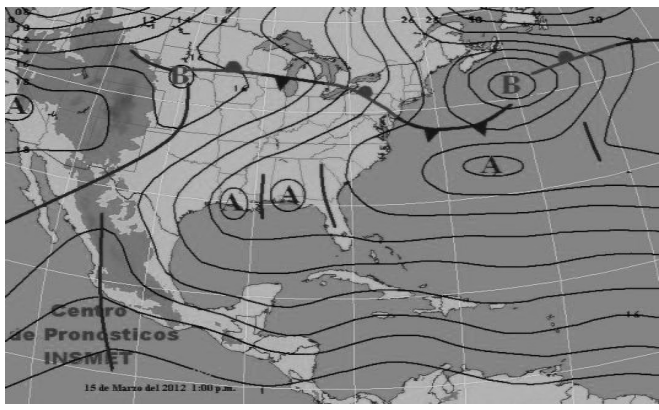

Figura 1 - Viento predominante del primer cuadrante.

$$
\begin{aligned}
Z i c_{i}= & Z i c_{i-1}+\left[(1+2 A) \frac{H_{i-1}}{\rho_{i-1} C_{p} \gamma_{i-1} Z i c_{i-1}}+\right. \\
& \left.+2 B \frac{u_{*_{i-1}}^{3} T_{r e f}}{\gamma_{i-1} g \operatorname{Zic}_{i-1}{ }^{2}}\right] \Delta t ; \quad i=2,3,4 \ldots \ldots
\end{aligned}
$$

Donde $\mathrm{H}_{\mathrm{i}-1}, \rho_{i-1}, \gamma_{i-1}, \mathrm{~T}_{\mathrm{i}-1}, u_{* i-1}$, son los valores de las variables en el instante de tiempo $t=(i-1) \Delta t$. Considerando $t_{o}=0$ como el momento a partir de la cual el flujo de calor y consecuentemente, (Zic) empiezan a crecer, se establecieron las condiciones iniciales siguientes: $\operatorname{Zic}(0)=0$ y $\mathrm{H}(0)=0$. El valor inicial de $\left(Z i c_{1}\right)$ se tomó igual a $50 \mathrm{~m}$.

\subsection{Clasificación de los Tipos de Situaciones Sinópticas (TSS)}

A continuación, se realiza una breve síntesis de los tipos de situaciones sinópticas (TSS), para una mejor comprensión de los resultados (Lapinel, 1988):

1) Cercana influencia del Anticiclón del Atlántico (AA).

TSS I. Cercana influencia del anticiclón del atlántico (Azores-Bermudas), el cual constituye el principal centro de acción de nuestra Área y es sin duda, el sistema sinóptico que con mayor frecuencia influye sobre Cuba. Se considera este tipo; cuando el anticiclón penetra en forma de cuña sobre Cuba, llegando a cubrir el Golfo de México y el sureste (SE) de los Estados Unidos (EU). El viento predominante asociado al sistema puede ser del primero (del Norte al Este) o del segundo cuadrante (del Este al Sur), lo que da origen a los subtipos: predominio de vientos del primer cuadrante (la), (Figura 1) y predominio de vientos del segundo cuadrante (Ib), (Figura 2).

\section{2) Flujo extendido (AE).}

TSS II. Se corresponden con aquellas configuraciones del campo bárico, que tienen lugar cuando la región central del anticiclón del atlántico se aleja sensiblemente de las áreas,

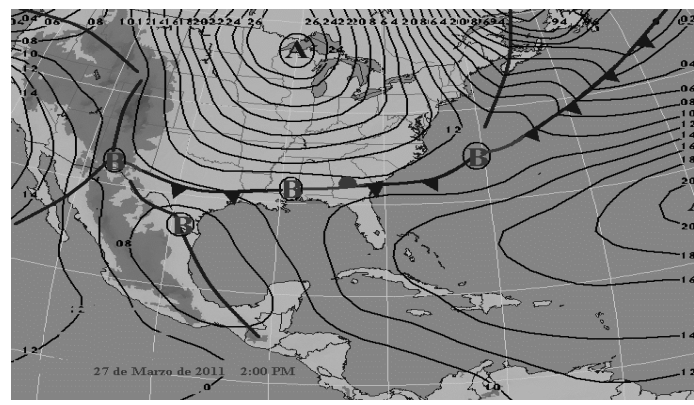

Figura 2 - Viento predominante del segundo cuadrante. 


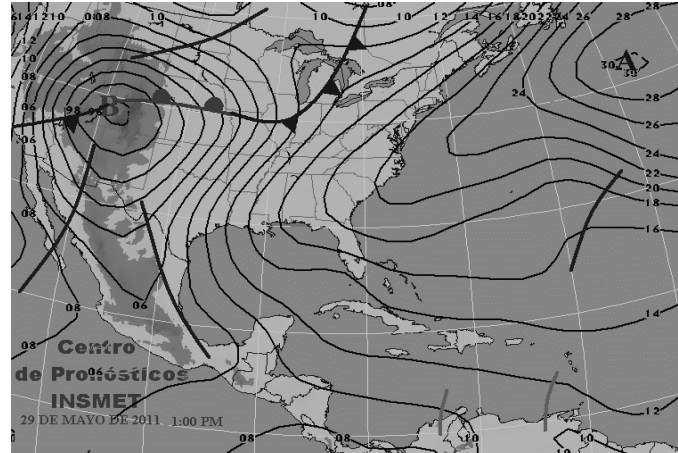

Figura 3 - Flujo extendido no perturbado.

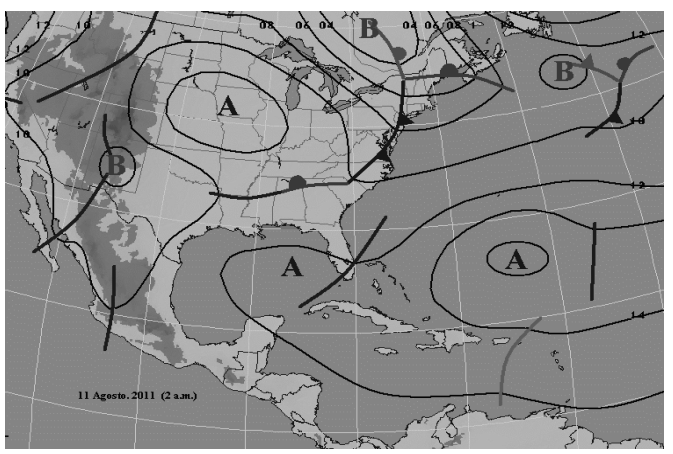

Figura 5 - Débil influencia anticiclónica.

permitiendo una mayor modificación de sus características iniciales, producto de una más prolongada interacción OcéanoAtmósfera (flujo extendido). Se consideran dos subtipos: flujo extendido no perturbado (IIa), (Figura 3 ) y el que corresponde a la influencia del sector de divergencia de las ondas u hondonadas (IIb), (Figura 4).

3) Débil gradiente barométrico (GD).

TSS III. Esta situación sinóptica, se relaciona con las configuraciones del campo bárico, que expresan una débil influencia anticiclónica o la existencia de hondonadas, vaguadas o sistemas de bajas en regiones adyacentes a las áreas en estudio cercanas, por lo que las isóbaras se muestran de una manera espaciada, predominando un débil gradiente barométrico (Figura 5).

\section{4) Situaciones ciclónicas (SC).}

TSS IV. Se relaciona con el desplazamiento cercano o sobre el territorio nacional de situaciones ciclónicas, tales como depresiones, perturbaciones o huracanes (Figura 6).

5) Zonas de convergencia en el flujo horizontal (ZC).

TSS V. En este tipo de situaciones sinópticas se agrupan las perturbaciones ondulatorias en el flujo básico de superficie,

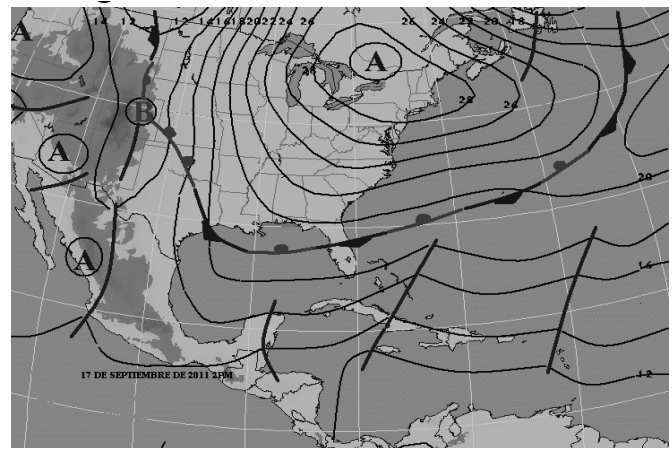

Figura 4 - Influencia del sector de divergencia de las ondas u hondonadas.

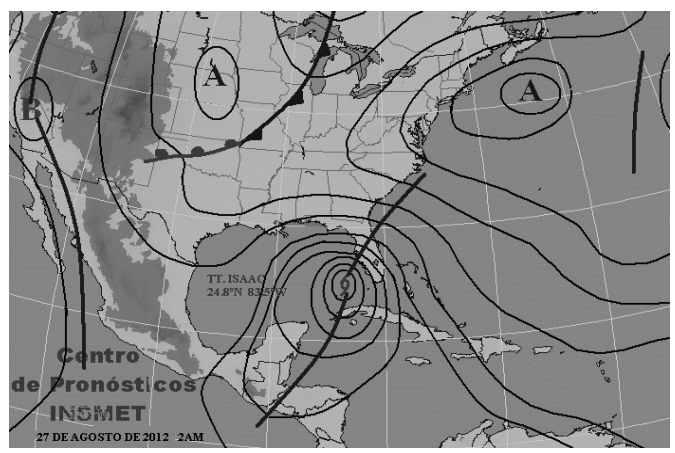

Figura 6 - Situaciones ciclónicas.

que influyen con sus áreas de convergencia asociadas sobre Cuba y zonas adyacentes. Existen dos subtipos: El que comprende ondas y hondonadas, que se generan y propagan en el flujo del este (Va), (Figura 7) y que consideran extensiones meridionales de vaguadas de latitudes medias, líneas de cizalladura entre dos altas, bajas frías (Vb), (Figura 8).

6) Bajas extratropicales (BE).

TSS VI. Se incluyen en este tipo las bajas extratropicales, que por regla general se originan en zonas troposféricas de intensa baroclinidad (frentes Ártico y Polar), resultantes de ondas de grandes dimensiones. Ocasionalmente tienen su origen en el Golfo de México o regiones cercanas a Cuba. Existen cuatro subtipos, según la forma de influencia sobre el territorio nacional: Influencia muy cercana o directa de la baja. Sector cálido (VIa) (Figura 9), Influencia muy cercana o directa de la baja. Sector frío (VIb) (Figura 10), Zona anterior al frente. Con hondonada pre-frontal (VIc) (Figura 11) y Zona anterior al frente. Sin hondonada pre-frontal (VId) (Figura 12).

\section{7) Zonas frontales (ZF).}

TSS VII. Se relaciona con los frentes fríos. A los efectos de la clasificación y en consideración con la estructura de los frentes, se clasifican o dividen en cuatro subtipos: El frente frío 


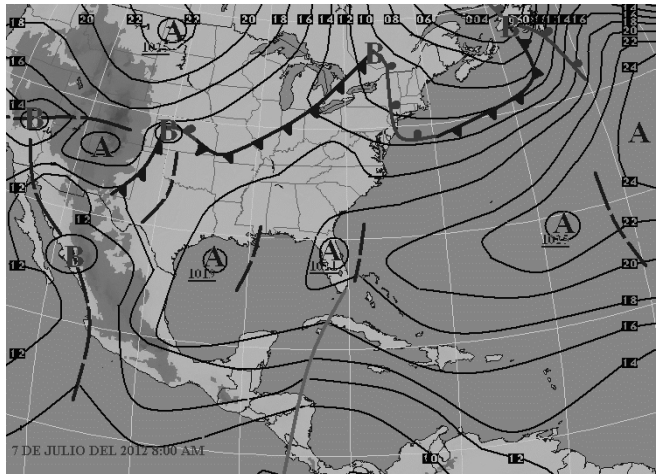

Figura 7 - Flujo del este.

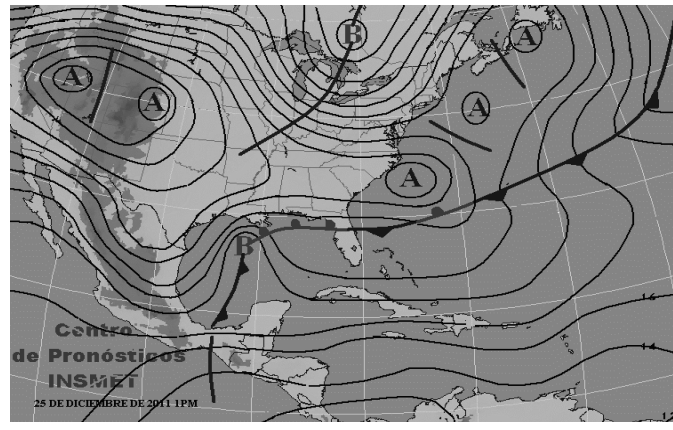

Figura 9 - Influencia muy cercana o directa de la baja. Sector cálido..

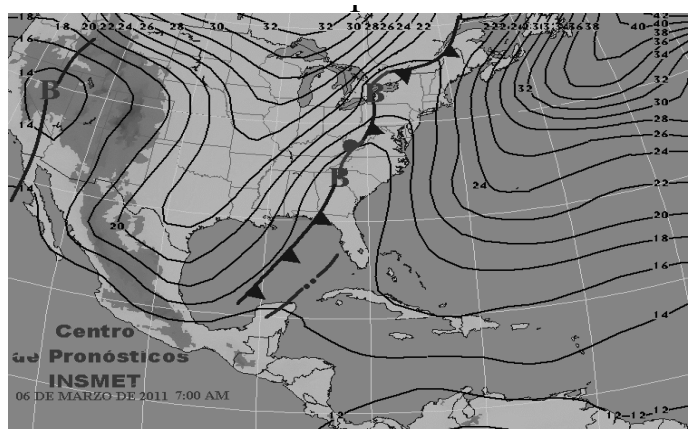

Figura 11 - Zona anterior al frente. Con hondonada pre-frontal.

clásico (VIla) (Figura 13), el frente frío revesino (VIIb) (Figura 14), el frente casi estacionario (VIIc) (Figura 15), y el frente disipándose (Vlld) (Figura 16).

8) Anticiclones continentales migratorios (AM).

TSS VIII. Se asocia al anticiclón continental polar de poco espesor, considerándose su profundidad en el orden de 1,5 a 3,0 km, aunque en ocasiones puede ser superior. Se consideran tres subtipos: Cuando el centro del anticiclón continental se encuentra en la parte central del continente (VIIla) (Figura 17). El centro anticiclón se encuentra al sur de los Estados Unidos y el Golfo de México (VIIIb) (Figura 18) y cuando se encuentra sobre el Océano Atlántico al este de los Estados Unidos (VIIIc) (Figura 19).

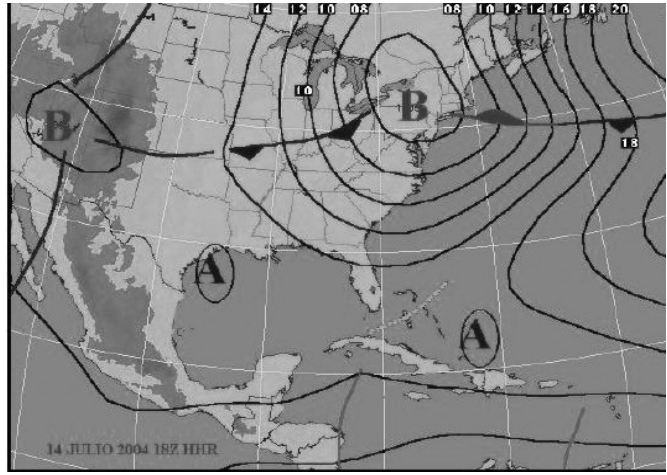

Figura 8 - Vaguadas de latitudes medias.

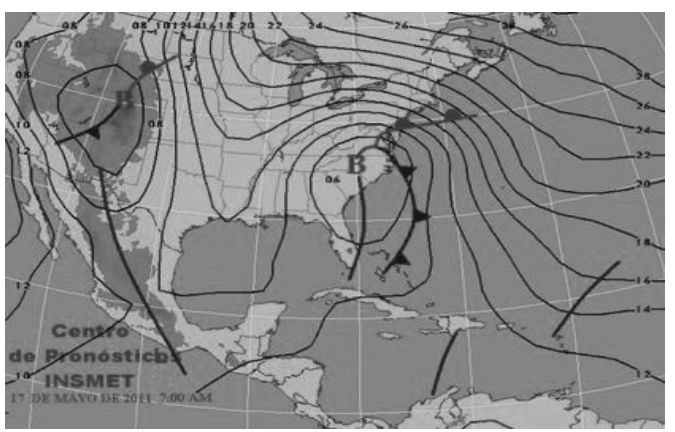

Figura 10 - Influencia muy cercana o directa de la baja. Sector frío.

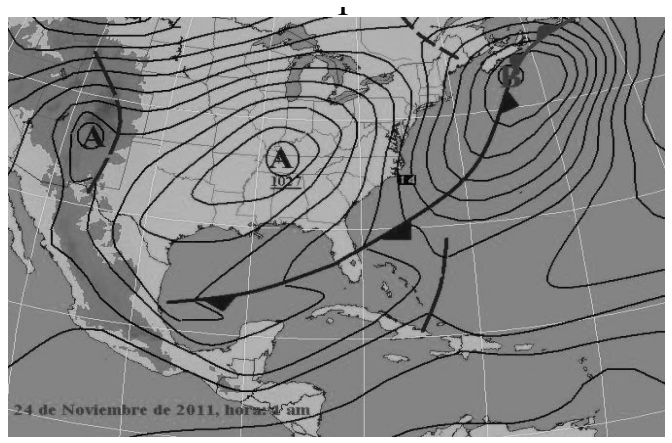

Figura 12 - Zona anterior al frente. Sin hondonada pre-frontal.

9) Otras situaciones (indeterminadas)

TSS IX. No es posible su identificación (Figura 20).

\subsection{Tratamiento estadístico de los datos}

En esta investigación, se comenzó con un estudio descriptivo de todos los parámetros que intervienen, realizándose además pruebas de bondad de ajuste para el chequeo del supuesto de normalidad, así como la prueba de las rachas para chequear la aleatoriedad en las muestras obtenidas. El resultado de estos análisis arrojo el no cumplimiento de los supuestos de normalidad y aleatoriedad; es por ello que se determinó aplicar las pruebas de la estadística no paramétrica, que son menos restrictivas y más eficientes en estas situaciones. 


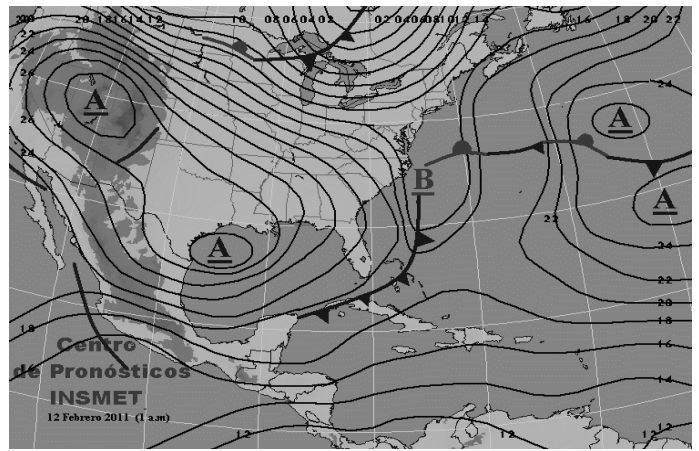

Figura 13 - Frente frío clásico.

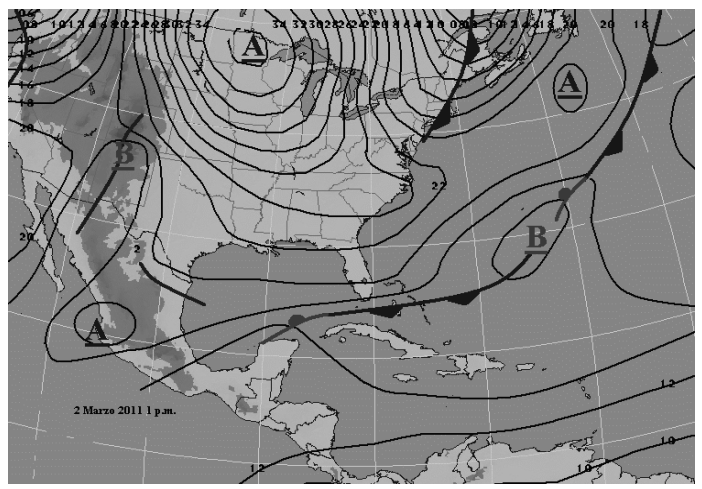

Figura 15 - Frente casi estacionario.

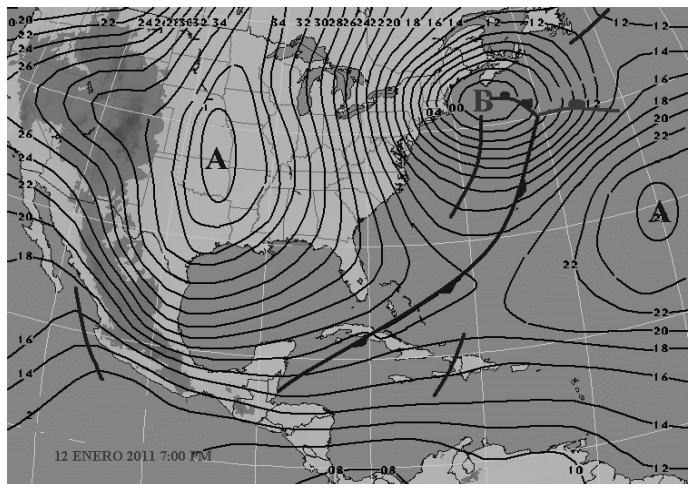

Figura 17 - Anticiclón continental en la parte central del continente.

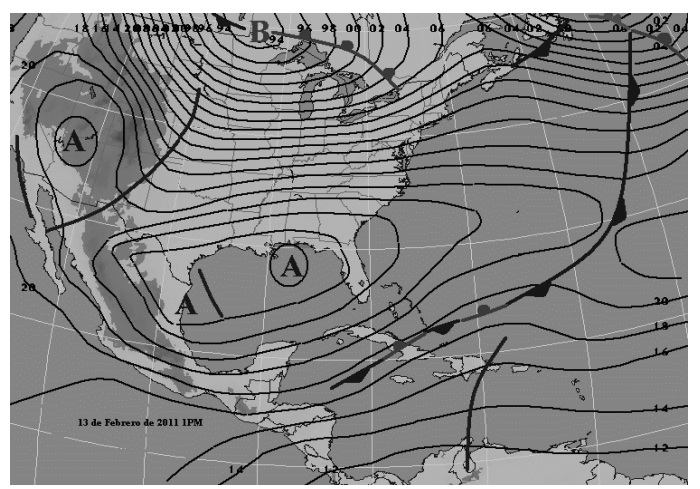

Figura 19 - Anticiclón sobre el Océano Atlántico al este de los Estados Unidos.

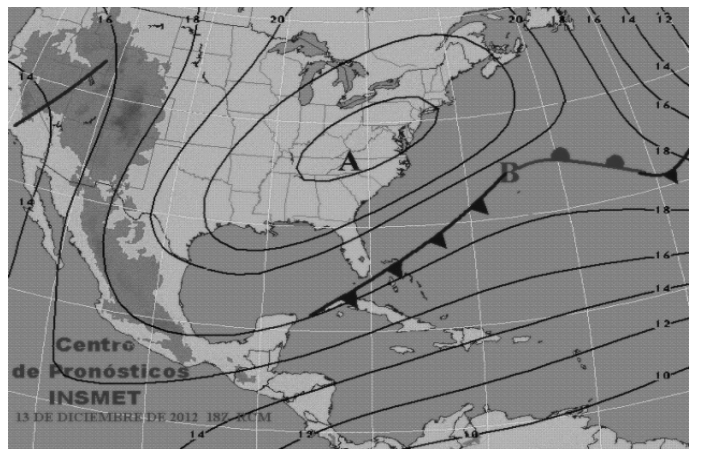

Figura 14 - Frente frío revesino.

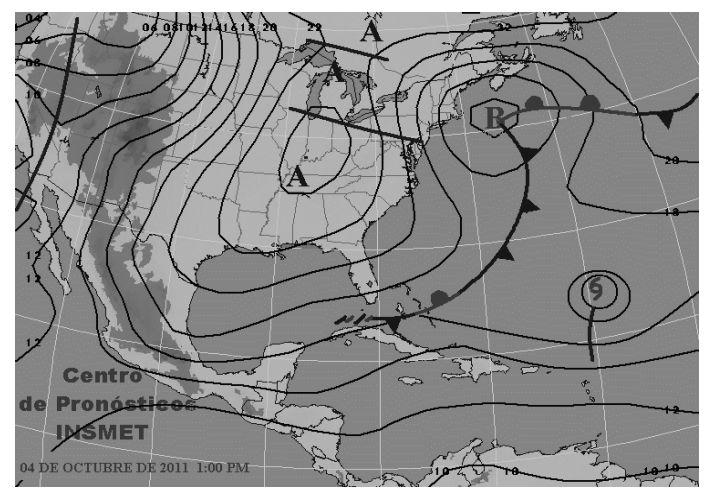

Figura 16 - Frente disipándose.

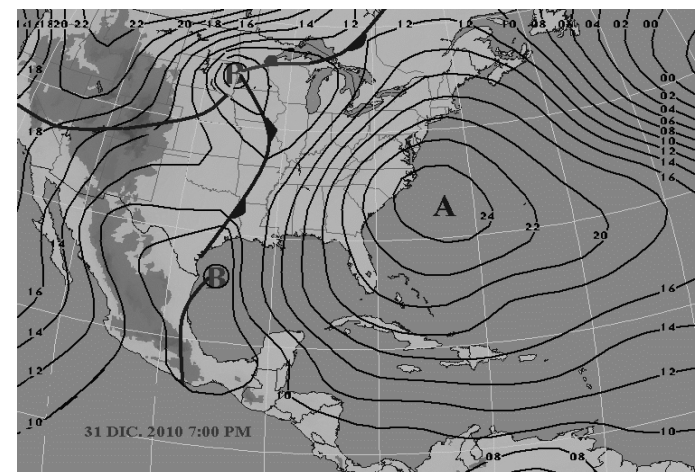

Figura 18 - Anticiclón se encuentra al sur de los Estados Unidos y el Golfo de México.

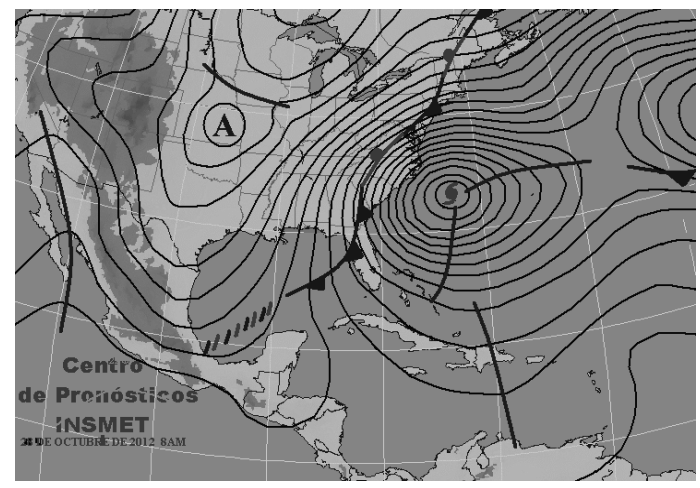

Figura 20 - Indeterminadas. 
El análisis de las relaciones existentes, entre los valores calculados de la altura de la capa de mezcla atmosférica para la ciudad de Pinar del Río en los horarios 7, 13, 16 y 17, se realizó por el método no paramétrico para $\mathrm{K}$ muestras relacionadas, mediante la prueba de (Friedman, 1937). Esta prueba sirve para comparar j promedios poblacionales cuando se trabaja con muestras relacionadas, la intención es averiguar si los promedios de esos tratamientos o muestras son o no iguales. Se aplico además la prueba no paramétrica de (Wilcoxon, 1945), la cual sirve para contrastar la hipótesis de igualdad de medianas, que es un estadístico más flexible y no se ve afectado por valores extremos. Esta es equivalente a la prueba t para poblaciones normales y se refiere a diseños antes después.

\subsection{Métodos y herramientas utilizadas para el procesamiento de los resultados}

Los algoritmos de cálculos empleados para obtener la estabilidad atmosférica y altura de la capa de mezcla atmosférica durante condiciones convectivas y estables, fueron implementados en el software MLHASC (González, 2013), empleando el lenguaje de programación Fortran 95 y los gráficos fueron realizados con Microsoft Excel. El procesamiento y análisis estadístico de los datos, se realizó mediante el software estadístico SPSS versión 15.0. Los datos meteorológicos de superficie: dirección del viento (DD), fuerza del viento (FF), humedad relativa $(\mathrm{Hr})$, temperatura de bulbo seco $\left(T_{\text {ref }}\right)$, nubosidad $(\mathrm{N})$ y presión a nivel de estación $(\mathrm{Pe})$, se obtuvieron de la estación meteorológica de la ciudad de Pinar del Río, perteneciente al Centro Meteorológico Provincial de Pinar del Río del Instituto de Meteorología del Ministerio de Ciencia, Tecnología y Medio Ambiente de Cuba.

\section{RESULTADOS Y DISCUSIÓN}

El estudio permitió obtener la variación horaria y mensual de la estabilidad atmosférica y de la altura promedio de la capa de mezcla atmosférica, así como la relación entre los valores de estabilidad atmosférica y altura de la capa de mezcla con los TSS. También se mostró la variación mensual a las 7 , 13, 16 y 17 horas de las dos variables en la ciudad de Pinar del Río, utilizando una serie de datos meteorológicos desde el 2006 hasta el 2010.

\subsection{Comportamiento de la Estabilidad Atmosférica (EA)}

El estudio de la variación horaria de la EA para la ciudad de Pinar del Río se muestra en la Figura 21, se observa que en las horas nocturnas solo ocurren las categorías muy estable (ME), estable (E), ligeramente estable (LE) y neutra $(\mathrm{N})$, siendo la categoría muy estable, con 1600 casos la de mayor ocurrencia, las demás categorías no alcanzaron los 200 casos de ocurrencia para el período analizado de cinco años. Este comportamiento se debió a que en la madrugada, se presentó la mayor cantidad de días con cielos despejados y con poca nubosidad y velocidades del viento con valores que, generalmente, no sobrepasaron $2 \mathrm{~m} / \mathrm{s}$, esto hace que se favorezca el proceso de irradiación terrestre, siendo el gradiente vertical de temperatura mayor que el gradiente adiabático seco, dando lugar a la ocurrencia de la categoría muy estable. En las horas diurnas sucede lo contrario, solo ocurren las categorías muy estable (ME), neutra $(\mathrm{N})$, ligeramente inestable (LI), inestable (I) y muy inestable (MI). Donde está última categoría presentó la mayor cantidad de casos, fundamentalmente de las 9 a las 17 horas, las demás categorías no alcanzaron los 300 casos de ocurrencia en todo el periodo analizado y solo ocurrieron en las 24 horas, las categorías muy estable y neutra. En el horario diurno el Sol provoca un aumento gradual de la temperatura y por consiguiente de la turbulencia, lo que unido a una mayor presencia de días despejados y con poca nubosidad, hace que el gradiente vertical de temperatura sea menor que el gradiente adiabático seco, generando la inestabilidad atmosférica a lo largo del día, donde la categoría muy inestable ejerce un mayor predominio.

La variación mensual de la EA en la ciudad de Pinar del Río se muestra en la Figura 22, donde se observa que las categorías muy estable (ME) y muy inestable (MI), tienen una proporción muy similar en la cantidad de casos, debido a que la variación mensual, constituye el promedio de la variación del ciclo diurno. La mayor cantidad de casos de ocurrencias corresponden a la categoría muy estable, siendo más marcada en los meses invernales, provocado por una menor cantidad de horas de luz solar. En los meses de verano disminuye su ocurrencia, debido al comportamiento estacional, donde la categoría muy inestable alcanzó la mayor cantidad de casos, observándose una transición más rápida de la categoría muy estable a la muy inestable en estos meses, lo cual obedece a una mayor incidencia de la irradiación y duración de las horas de luz solar, provocando un aumento de la temperatura y por consiguiente de la turbulencia atmosférica. Por ello las demás categoría tienen menor presencia en los meses de verano de junio a septiembre y mayor ocurrencia de casos en meses de enero a mayo y de octubre a diciembre, siguiendo estas categorías un comportamiento muy similar en el período evaluado.

La variación mensual de la EA en la ciudad de Pinar del Río a las 7 horas se muestra en la Figura 23, observándose que solo ocurren en todo el año las categorías: muy estable (ME), estable (E) y ligeramente estable (LE), siendo la categoría muy estable la que presentó mayor cantidad de casos de ocurrencias, sobrepasando los 130 casos en la mayoría de los meses, debido a que el sol se encuentra por debajo del horizonte y la atmósfera 


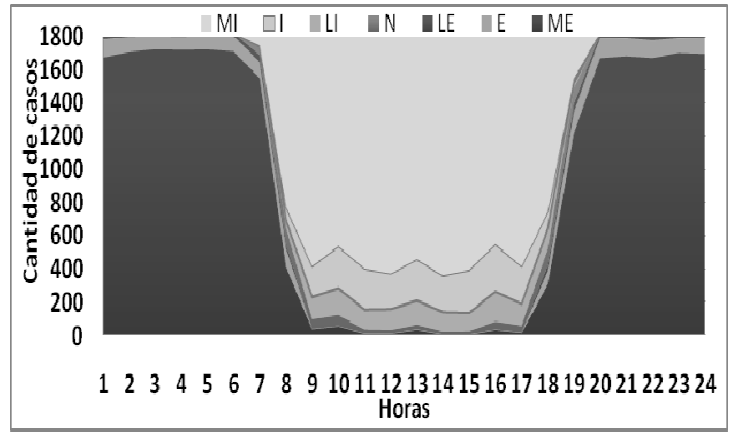

Figura 21 - Variación horaria de la EA en la ciudad de Pinar del Río.

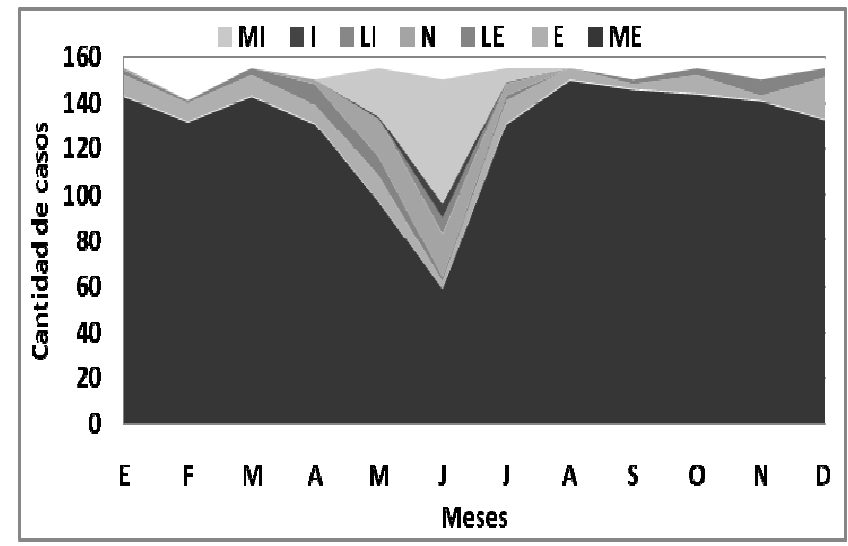

Figura 23 - Variación mensual de la EA a las 7 horas en la ciudad de Pinar del Río.

tiene un comportamiento estable, provocado por el proceso de irradiación terrestre, el cual se intensifica a lo largo de la noche. En los meses de mayo y junio disminuyó el comportamiento de la categoría muy estable, por el aumento de la ocurrencia de la categoría muy inestable (MI), la que fue superior en el último mes con 54 casos de ocurrencia, esto obedece al comportamiento estacional, teniendo presencia el sol en estos meses, rompiendo así con la EA creada en la noche. Las demás categorías: neutra $(\mathrm{N})$, ligeramente inestable (LI) e inestable (I) presentaron un comportamiento muy similar en todo el período evaluado.

El comportamiento de la EA a las 13 horas en la ciudad de Pinar del Río (Figura 24), muestra que solo ocurrieron en todo el año las categorías: ligeramente inestable (LI), inestable (I) y muy inestable (MI), donde está ultima alcanzó la mayor cantidad de casos de ocurrencia, presentando sus máximos valores en los meses de julio y agosto, siendo superior en este último mes, con 142 casos. Este comportamiento obedece al ciclo diario y estacional, a esta hora el sol tiene la mayor altura sobre el horizonte, lo que produce elevadas temperaturas, generando así un aumento significativo de la turbulencia, fundamentalmente en los meses de verano, debido a una mayor intensidad de

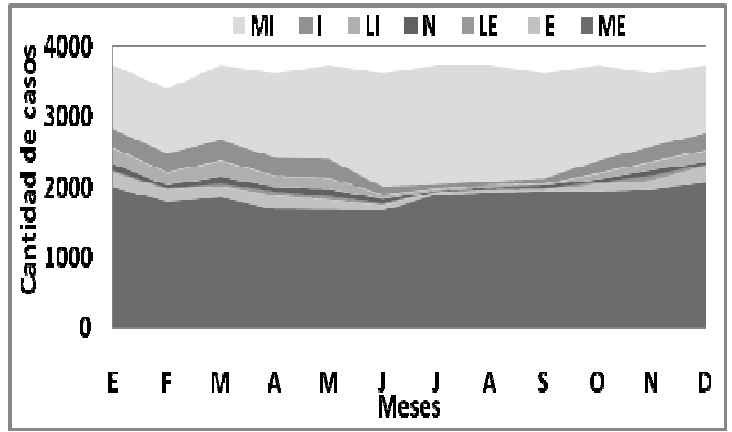

Figura 22 - Variación mensual de la EA en la ciudad de Pinar del Río.

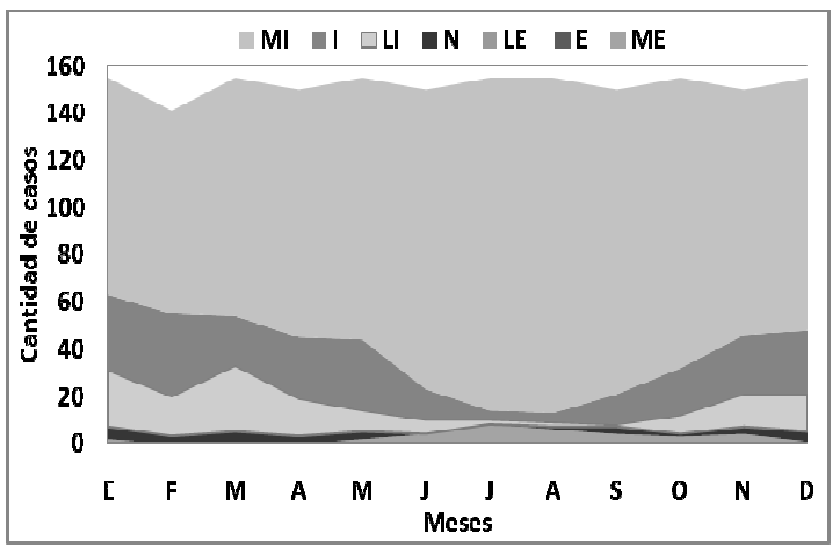

Figura 24 - Variación mensual de la EA a las 13 horas en la ciudad de Pinar del Río.

la irradiación y de la luz solar. Las demás categorías que ocurrieron: la muy estable (ME) y neutra $(\mathrm{N})$, tuvieron pocos casos de ocurrencia.

A las 16 y 17 horas la variación mensual de la EA en la ciudad de Pinar del Río (Figuras 25 y 26), presentó un comportamiento muy similar, ocurriendo en todo el año las categorías: neutra $(\mathrm{N})$, ligeramente inestable (LI), inestable (I) y muy inestable (ME), donde está ultima mostró la mayor cantidad de casos de ocurrencia, presentando su máximo valor en el mes de julio (144 casos) a las 17 horas. Este comportamiento obedece al ciclo diario y estacional, la turbulencia atmosférica va aumentado a lo largo del día, debido a la incidencia de la irradiación solar sobre la superficie terrestre, esto provoca un aumento gradual de la temperatura sobre está, la que se encarga de calentar a la atmósfera, provocando que en estos horarios exista una marcada inestabilidad atmosférica, la que se incrementa significativamente en los meses de verano, fundamentalmente cuando existe poca fuerza del viento. Las restantes categorías que ocurrieron tuvieron valores muy inferiores y la categoría muy estable (ME) no ocurrió en todo el período evaluado. 


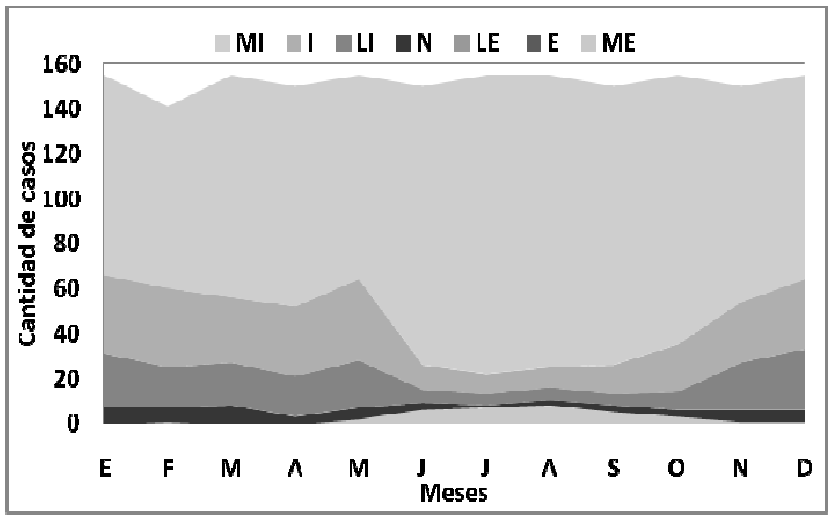

Figura 25 - Variación mensual de la EA a las 16 horas en la ciudad de Pinar del Río.

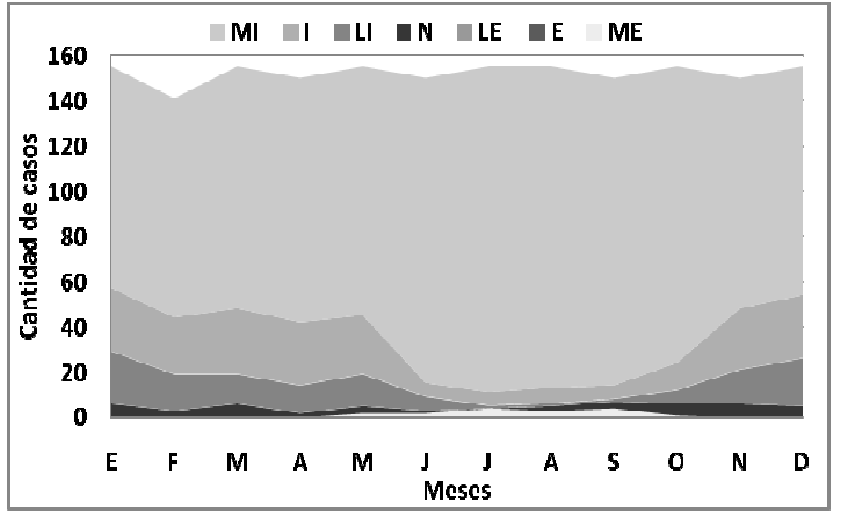

Figura 26 - Variación mensual de la EA a las 17 horas en la ciudad de Pinar del Río.

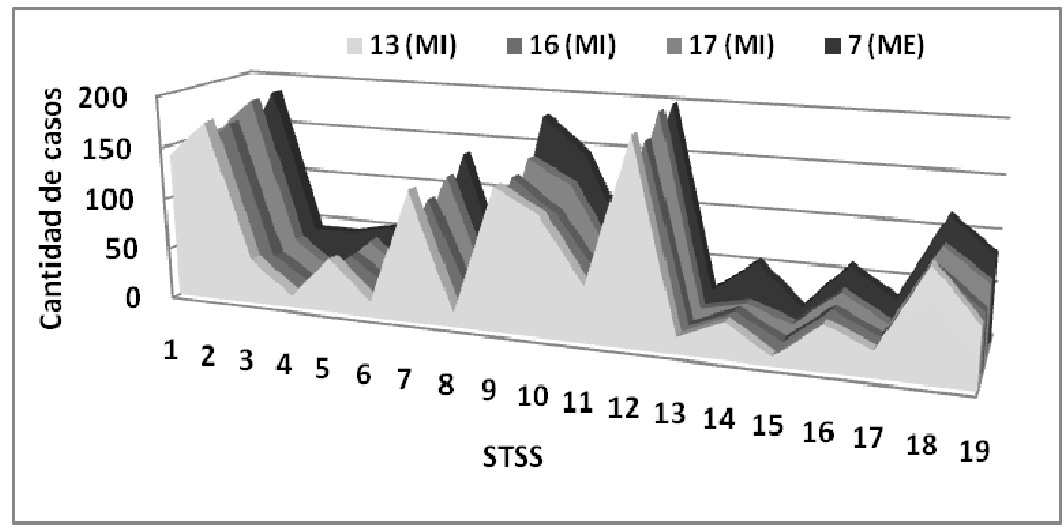

Figura 27 - Estabilidad atmosférica de mayor ocurrencia para cada STSS, en la ciudad de Pinar del Río, a las 7, 13, 16 y 17 horas.

\subsection{Relación entre los valores de estabilidad atmosférica y los SS}

La EA juega un papel primordial en la dispersión y transporte de contaminantes y su relación con los diferentes tipos de situaciones sinópticas (TSS), influyen en su comportamiento. Esta se manifiesta de diversas maneras en el transcurso del día, debido precisamente a las condiciones imperantes en el clima. Existen cuatro horarios que toman una singular importancia en estudios de contaminación: 7, 13, 16 y 17 horas. Como se puede observar en la Tabla 2 se muestra el comportamiento de la EA para los TSS, desglosado por sus subtipos (STSS). Apreciándose que a las 7 horas, existen condiciones desfavorables para la dispersión de los contaminantes, debido a las condiciones estables presente en la atmósfera. En los demás horarios analizados, existen condiciones favorables para su dispersión, por la alta ocurrencia de inestabilidad atmosférica.

El estudio mostró la categoría de EA de mayor ocurrencia en las horas analizadas y que TSS fue predominante
(Tabla 2). A las 7 horas fue la muy estable (ME) bajo el predominio del TSS VIII (anticiclones migratorios), a medida que el anticiclón se desplaza desde Norte América, transita sobre el Golfo de México, se caracteriza por una disminución de la fuerza del viento y un giro del rumbo más al Este, cuando se interna en el Océano Atlántico, continúa disminuyendo la fuerza del viento y el rumbo Este es más marcado, esto hace que su alta influencia sobre Cuba, contribuya a la ocurrencia de categoría muy estable, unido a la poca presencia del sol a esta hora. En los demás horarios de interés el TSS de mayor ocurrencia fue la I (anticiclón del atlántico), el cual constituye el principal centro de acción y el sistema sinóptico que con mayor frecuencia influye sobre Cuba, se caracteriza por vientos predominantes de región Este de poca fuerza, unido al aumento de la turbulencia atmosférica, debido a que las mayores temperaturas se registran en las horas de la tarde $(13,16$ y 17), hacen que la atmósfera se comporte muy inestable (MI). En los horarios analizados la mayor ocurrencia se presentó bajo la influencia de Zonas típicas del anticiclón extendido sin hondonada, subtipo 12 (Figura 27). 
Tabla 2 - Estabilidad atmosférica (EA) de mayor ocurrencia para cada TSS en la ciudad de pinar del Río en los horarios 7, 13, 16 y 17 en el período 2006-2010.

\begin{tabular}{|c|c|c|c|c|c|}
\hline \multirow[b]{2}{*}{ TSS } & \multirow[b]{2}{*}{ STSS } & \multicolumn{4}{|c|}{ Horarios } \\
\hline & & 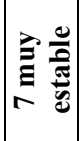 & 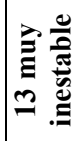 & 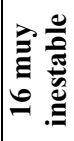 & 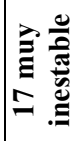 \\
\hline \multirow{2}{*}{$\begin{array}{l}\text { I. Anticiclón } \\
\text { del Atlántico }\end{array}$} & 1. Predominio de vientos del primer cuadrante & 117 & 141 & 132 & 149 \\
\hline & \begin{tabular}{|l}
$\begin{array}{l}\text { 2. Predominio de vientos del } \\
\text { cuadrante }\end{array}$
\end{tabular} & 185 & 176 & 168 & 184 \\
\hline \multirow{4}{*}{$\begin{array}{c}\text { VI. Baja } \\
\text { Extratropical }\end{array}$} & $\begin{array}{l}\text { 3. Influencia muy cercana o directa de la baja. } \\
\text { Sector cálido }\end{array}$ & 44 & 44 & 37 & 43 \\
\hline & $\begin{array}{l}\text { 4. Influencia muy cercana o directa de la baja. } \\
\text { Sector frío }\end{array}$ & 44 & 12 & 12 & 14 \\
\hline & $\begin{array}{l}\text { 5. Zona anterior al frente. Con hondonada pre- } \\
\text { frontal }\end{array}$ & 53 & 53 & 45 & 49 \\
\hline & $\begin{array}{l}\text { 6. Zona anterior al frente. Sin hondonada pre- } \\
\text { frontal }\end{array}$ & 17 & 15 & 18 & 18 \\
\hline \multirow{2}{*}{$\begin{array}{c}\text { V. Zonas de } \\
\text { Convergencia }\end{array}$} & 7. En el flujo del Este & 136 & 127 & 109 & 122 \\
\hline & 8. Otros & 15 & 14 & 14 & 14 \\
\hline \multirow{3}{*}{$\begin{array}{l}\text { VIII. } \\
\text { Anticiclones } \\
\text { Migratorios }\end{array}$} & 9. Sobre el continente & 179 & 137 & 137 & 146 \\
\hline & 10. Al Sur de los EU y el Golfo de México & 145 & 115 & 107 & 124 \\
\hline & $\begin{array}{l}\text { 11. Sobre el Océano Atlántico al este de los } \\
\text { EU }\end{array}$ & 62 & 53 & 52 & 57 \\
\hline \multirow{2}{*}{$\begin{array}{l}\text { II. Anticiclón } \\
\text { Extendido }\end{array}$} & $\begin{array}{l}\text { 12. Zonas típicas del Anticiclón extendido (sin } \\
\text { hondonada) }\end{array}$ & 199 & 193 & 180 & 199 \\
\hline & $\begin{array}{l}\text { 13. Zonas típicas del Anticiclón extendido } \\
\text { (región divergente) }\end{array}$ & 23 & 16 & 14 & 17 \\
\hline \multirow{4}{*}{$\begin{array}{l}\text { VII. Zonas } \\
\text { Frontales }\end{array}$} & 14. Frente frío clásico & 55 & 35 & 32 & 27 \\
\hline & 15. Frente frío revesino & 15 & 9 & 9 & 12 \\
\hline & 16. Frente estacionario & 60 & 43 & 43 & 47 \\
\hline & 17. Frente disipándose & 33 & 25 & 23 & 23 \\
\hline $\begin{array}{l}\text { III. Gradiente } \\
\text { Débil }\end{array}$ & $\begin{array}{l}\text { 18. Generalmente asociado a ondas } \mathrm{u} \\
\text { hondonadas. }\end{array}$ & 115 & 104 & 89 & 98 \\
\hline $\begin{array}{l}\text { IV. Situaciones } \\
\text { Ciclónicas }\end{array}$ & $\begin{array}{l}\text { 19. Depresiones ciclónicas, perturbaciones, } \\
\text { huracanes. }\end{array}$ & 83 & 56 & 55 & 68 \\
\hline
\end{tabular}

\subsection{Comportamiento de la Altura Promedio de la Capa de Mezcla atmasférica (APCM)}

El estudio de la variación horaria de la altura promedio de la capa de mezcla (APCM) atmosférica en la ciudad de Pinar del Río se muestra en la Figura 28, observándose que en las horas nocturnas la altura no llega a $200 \mathrm{~m}$. Después de la puesta de sol, la superficie terrestre no recibe irradiancia solar, pero sí emite irradiancia en onda larga, consecuentemente, el suelo y el aire en contacto con él, así como las capas más bajas de la atmósfera, se van enfriando más rápidamente que el aire en las capas más altas. De esta forma se obtiene un aumento de la temperatura con la altura y, por tanto, una capa térmicamente estable en la parte inferior de la atmósfera donde el aire con estratificación estable tiende a suprimir la turbulencia. En el horario diurno ocurre el proceso inverso, en las primeras horas de la mañana la APCM atmosférica comienza a crecer, debido al aumento gradual de la temperatura y de la turbulencia atmosférica, provocado por la irradiancia solar, alcanzando su máximo valor muy próximo a los $1900 \mathrm{~m}$ a las 17 horas, fundamentalmente en días despejados y con poca nubosidad.

La variación mensual de la APCM atmosférica en la ciudad de Pinar del Río se muestra en Figura 29, observándose los menores valores de altura en los meses de enero, febrero, octubre, noviembre y diciembre, siendo inferior a $500 \mathrm{~m}$ en enero y diciembre y un poco superior en los demás meses mencionados, este comportamiento obedece al ciclo estacional, donde la cantidad de horas de luz e intensidad de la irradiación solar es menor, esto hace que las temperaturas no sean tan elevadas, disminuyendo así la turbulencia atmosférica. En los meses de marzo a septiembre, se experimenta un crecimiento de la duración de las horas de luz solar, esto hace que se incremente 


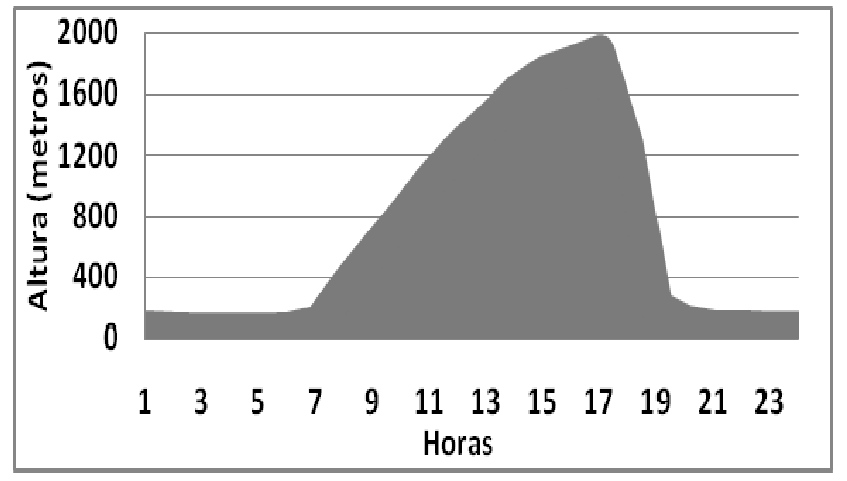

Figura 28 - Variación horaria de la APCM atmosférica en la ciudad de Pinar del Río.

la turbulencia atmosférica debido al aumento de las temperaturas, dando como resultado que la APCM atmosférica comience a crecer superando los $600 \mathrm{~m}$ en estos meses, alcanzando su máximo valor en mayo, muy próximo a los $750 \mathrm{~m}$ de altura.

El estudio de la variación mensual de la APCM atmosférica a las 7 y 13 horas para la ciudad de Pinar del Río se muestra en las Figuras 30 y 31. El estudio de la variación mensual de la APCM atmosférica a las 7 y 13 horas para la ciudad de Pinar del Río se muestra en las Figuras 30 y 31. A las 7 horas (Figura 30) solo existe presencia de la irradiación solar en mayo y junio, superándose los $200 \mathrm{~m}$ de altura en estos meses, siendo superior en mayo $(281 \mathrm{~m})$, debido al aumento de las temperaturas y consecuentemente de la turbulencia atmosférica. Los demás meses presentan valores inferiores a 50 $m$ excepto en enero, abril, julio y diciembre que son un poco superior a esta altura, este comportamiento se debe a una mayor estabilidad atmosférica.

La variación mensual de la APCM atmosférica a las 13 horas para la ciudad de Pinar del Río se ilustra en Figura 31, observándose que se experimenta un mayor crecimiento de

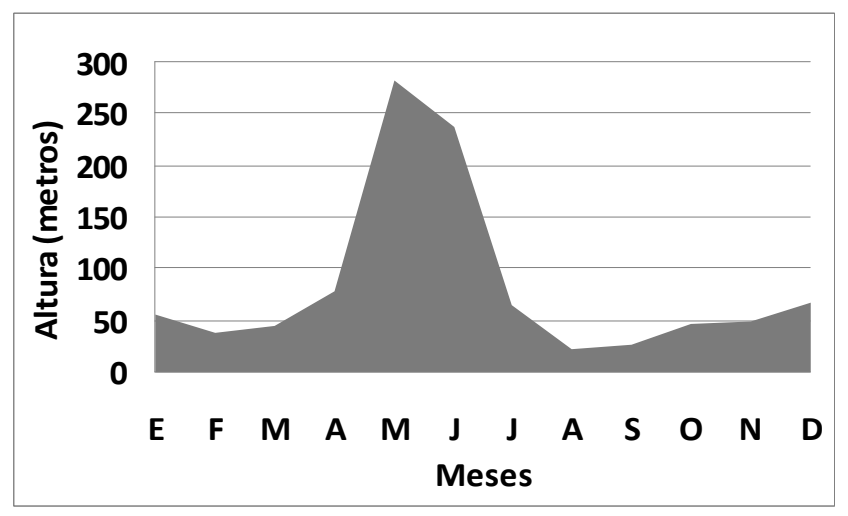

Figura 30 - Variación mensual de la APCM atmosférica a las 7 horas en la ciudad de Pinar del Río.de Pinar del Río.

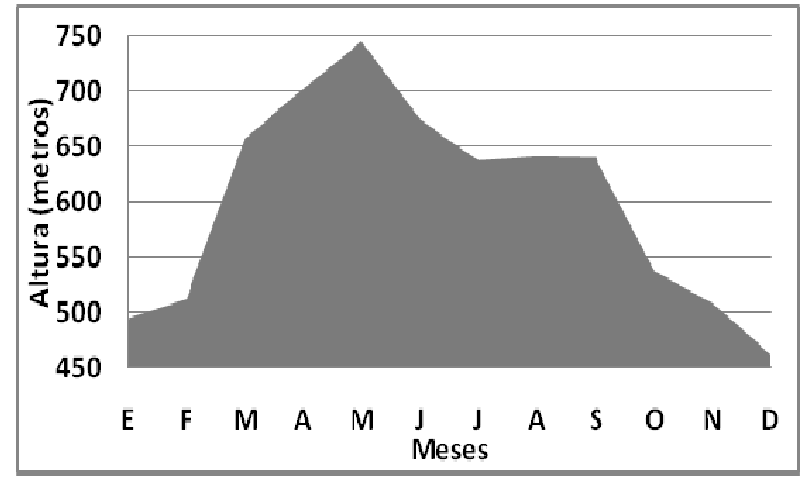

Figura 29 - Variación mensual de la APCM atmosférica en la ciudad de Pinar del Río.

la altura a esta hora, presentando sus máximos valores en los meses de abril y mayo, siendo máximo su comportamiento en este último mes con $1597 \mathrm{~m}$. El menor valor ocurrió en enero con $607 \mathrm{~m}$, los demás meses restantes superaron los $1200 \mathrm{~m}$ de altura. A esta hora la atmósfera presenta mayor turbulencia que a las 7 horas, provocado por el máximo de elevación solar sobre el horizonte, aumentando considerablemente la temperatura, fundamentalmente en los meses de verano, debido al ciclo estacional.

La variación mensual de la APCM atmosférica a las 16 y 17 horas para la ciudad de Pinar del Río (Figuras 32 y $33)$, muestra un comportamiento muy similar, presentando sus máximos valores en los meses de abril y mayo, estando muy próximo a los $2000 \mathrm{~m}$ de altura a las 16 horas, mientras que a las 17 horas sobrepasan este valor. La turbulencia atmosférica aumenta a lo largo del día, debido a la incidencia de la irradiación solar sobre la superficie terrestre, lo que genera un aumento progresivo de la temperatura ambiental, provocando que en estos horarios se experimenten los mayores valores de altura de mezclamiento en el ciclo diurno, siendo máximo este

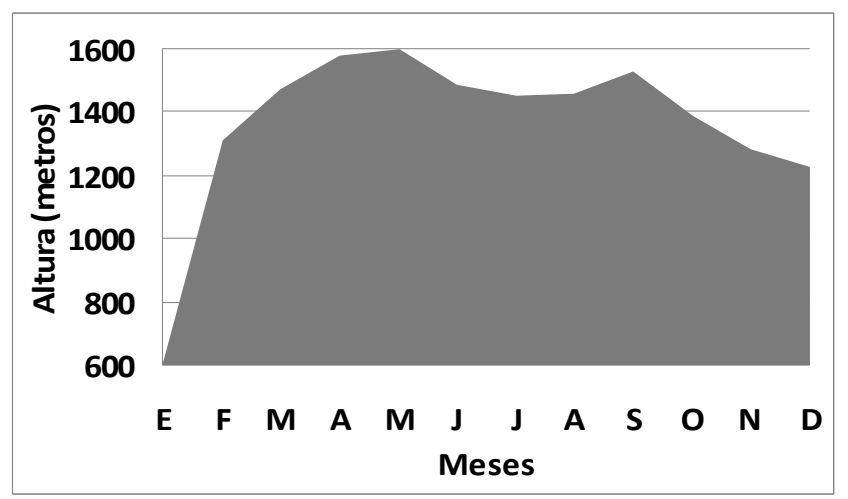

Figura 31 - Variación mensual de la APCM atmosférica a las 13 horas en la ciudad de Pinar del Río. 


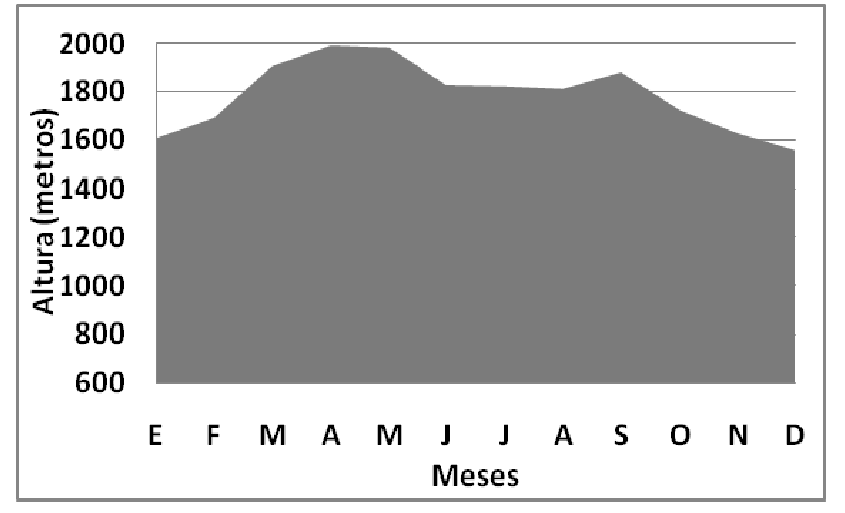

Figura 32 - Variación mensual de la APCM atmosférica a las 16 horas en la ciudad de Pinar del Río.

comportamiento a las 17 horas, principalmente cuando existe poca fuerza del viento.

\subsection{Relación entre los valores de altura promedio de la capa de mezcla y los TSS}

La relación que existe entre los valores de APCM con las diversas masas de aire asociadas a los diferentes tipos de situaciones sinópticas, tienen gran importancia para conocer el comportamiento de los contaminantes y determinar el nivel de impacto sobre el medio ambiente y los seres humanos. La altura de la capa de mezcla varía constantemente, existiendo horarios en que es de vital importancia conocer su comportamiento, atendiendo al régimen de trabajo de las fuentes, las que de forma general comienzan a las 7 de la mañana, presentándose condiciones desfavorables a esta hora para la dispersión de los contaminantes por la poca altura de mezclamiento. Los demás horarios de interés presentan mayores alturas de mezclamiento, favoreciendo así la dispersión de los contaminantes. En la Tabla 3 se muestra la correspondencia de la APCM con las distintas situaciones sinópticas en los horarios analizados.

El máximo valor de APCM atmosférica, ocurre bajo la presencia del TSS IV (situaciones ciclónicas) para los horarios 13 y 17, alcanzándose el máximo valor a las 17 horas, esto se debe a que la Ecuación 4, descrita en el trabajo, depende de la fuerza del viento y está se hace máxima bajo la influencia del TSS IV, además del incremento significativo de la turbulencia atmosférica que ocurre en estos horarios producto al aumento de la temperatura ambiente. A las 7 y 16 horas, el máximo ocurre bajo la influencia del TSS VI (baja extratropical), este TSS, también produce velocidades del viento fuerte, la poca altura de mezclamiento que se experimenta a las 7 horas, guarda una relación directa con la ausencia del sol en la mayor parte del año, excepto en los meses de verano. En el análisis de los subtipos se observó, que los mayores valores de

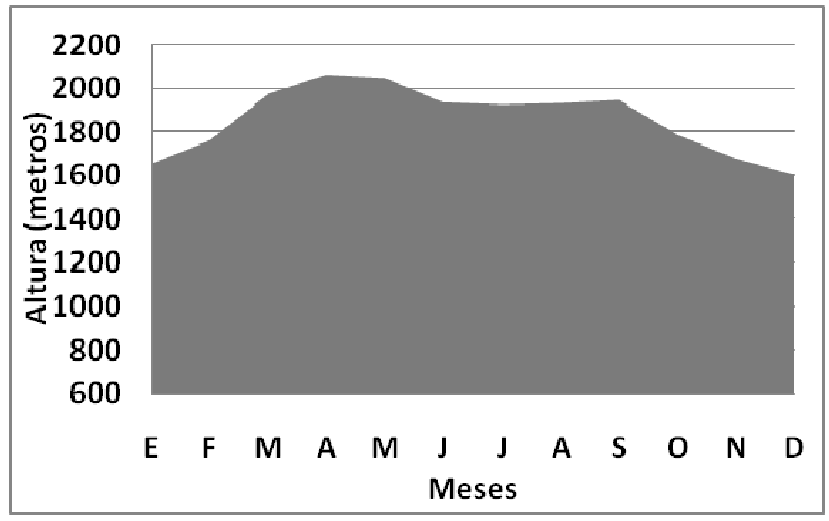

Figura 33 - Variación mensual de la APCM atmosférica a las 17 horas en la ciudad de Pinar del Río.

APCM atmosférica por horario se presentó para los subtipos: 6 (Zona anterior al frente. Sin hondonada pre-frontal) para las 7 horas con 257 m; 19 (Depresiones ciclónicas, perturbaciones, huracanes) para las 13 horas $(1554 \mathrm{~m})$ y 17 horas con (2036 m); 4 (Influencia muy cercana o directa de la baja. Sector frío) para las 16 horas (1935 m). En la Figura 34, se muestra el comportamiento de la APCM atmosférica para cada STSS, observándose que los mayores valores ocurrieron a las 17 horas para el período analizado.

\subsection{Análisis estadístico de la altura de la capa de mezcla atmosférica}

Se realizó la prueba no paramétrica de Friedman para la comparación de los valores de la APCM atmosférica en la ciudad de Pinar del Río, calculados para un período de cinco años (2006-2010) en las horas 7, 13, 16 y 17. Los resultados obtenidos de la aplicación de esta prueba para un nivel de significación del $5 \%$ arrojan diferencias significativas $(\mathrm{p}=$ $0,000)$ en la APCM atmosférica en los diferentes horarios analizados en la cuidad de Pinar del Río. Para investigar qué horarios inciden más significativamente en el comportamiento de esta variable se aplicó la prueba no paramétrica de Wilcoxon para dos muestras relacionadas, acompañada de la corrección de Bonferroni para controlar la tasa de error. Esta prueba permitió demostrar que existe diferencia en la APCM atmosférica entre todos los horarios, comparadas dos a dos, observándose que es a las 17 horas donde alcanza su mayor altura.

\section{CONCLUSIONES}

- La variación horaria de la estabilidad atmosférica, mostró que en las horas diurnas existen las mejores condiciones para la dispersión de los contaminantes atmosféricos, 
Tabla 3 - Altura promedio de la capa de mezcla en las zonas de estudio para cada TSS en los horarios 7, 13, 16 y 17 en el período $2006-2010$.

\begin{tabular}{|c|c|c|c|c|c|}
\hline \multirow{2}{*}{ TSS } & \multirow{2}{*}{ STSS } & \multicolumn{4}{|c|}{ Horarios } \\
\hline & & 7 & 13 & 16 & 17 \\
\hline \multirow{2}{*}{$\begin{array}{l}\text { I. Anticiclón del } \\
\text { Atlántico }\end{array}$} & $\begin{array}{l}\text { 1. Predominio de vientos del primer } \\
\text { cuadrante }\end{array}$ & 143 & 1485 & 1850 & 1944 \\
\hline & $\begin{array}{l}\text { 2. Predominio de vientos del segundo } \\
\text { cuadrante }\end{array}$ & 57 & 1467 & 1819 & 1910 \\
\hline \multirow{4}{*}{$\begin{array}{l}\text { VI. Baja } \\
\text { Extratropical }\end{array}$} & $\begin{array}{l}\text { 3. Influencia muy cercana o directa de la } \\
\text { baja. Sector cálido }\end{array}$ & 40 & 1385 & 1834 & 1897 \\
\hline & $\begin{array}{l}\text { 4. Influencia muy cercana o directa de la } \\
\text { baja. Sector frío }\end{array}$ & 6 & 1544 & 1935 & 1994 \\
\hline & $\begin{array}{l}\text { 5. Zona anterior al frente. } \\
\text { hondonada pre-frontal }\end{array}$ & 32 & 1309 & 1659 & 1768 \\
\hline & $\begin{array}{l}\begin{array}{l}\text { Zona anterior al frente. } \\
\text { hondonada pre-frontal }\end{array} \\
\text { hon }\end{array}$ & 257 & 1340 & 1693 & 1749 \\
\hline \multirow{2}{*}{$\begin{array}{l}\text { V. Zonas de } \\
\text { Convergencia }\end{array}$} & 7. En el flujo del Este & 114 & 1458 & 1769 & 1857 \\
\hline & 8. Otros & 22 & 1335 & 1850 & 1897 \\
\hline \multirow{3}{*}{$\begin{array}{l}\text { VIII. } \\
\text { Anticiclones } \\
\text { Migratorios }\end{array}$} & 9. Sobre el continente & 75 & 1394 & 1736 & 1800 \\
\hline & $\begin{array}{l}\text { 10. Al Sur de los EU y el Golfo de } \\
\text { México }\end{array}$ & 153 & 1458 & 1851 & 1903 \\
\hline & $\begin{array}{l}\text { 11. Sobre el Océano Atlántico al este de } \\
\text { los EU }\end{array}$ & 45 & 1353 & 1723 & 1778 \\
\hline \multirow{2}{*}{$\begin{array}{l}\text { II. Anticiclón } \\
\text { Extendido }\end{array}$} & $\begin{array}{l}\text { 12. Zonas típicas del Anticiclón } \\
\text { extendido (sin hondonada) }\end{array}$ & 94 & 1409 & 1804 & 1864 \\
\hline & $\begin{array}{l}\text { 13. Zonas típicas del Anticiclón } \\
\text { extendido (región divergente) }\end{array}$ & 44 & 1191 & 1546 & 1594 \\
\hline \multirow{4}{*}{$\begin{array}{l}\text { VII. Zonas } \\
\text { Frontales }\end{array}$} & 14. Frente frío clásico & 69 & 1302 & 1668 & 1721 \\
\hline & 15. Frente frío revesino & 55 & 1303 & 1649 & 1695 \\
\hline & 16. Frente estacionario & 19 & 1232 & 1616 & 1697 \\
\hline & 17. Frente disipándose & 11 & 1308 & 1682 & 1735 \\
\hline $\begin{array}{l}\text { III. Gradiente } \\
\text { Débil }\end{array}$ & $\begin{array}{l}\text { 18. Generalmente asociado a ondas } \mathrm{u} \\
\text { hondonadas. }\end{array}$ & 44 & 1436 & 1827 & 1885 \\
\hline $\begin{array}{l}\text { IV. Situaciones } \\
\text { Ciclónicas }\end{array}$ & $\begin{array}{l}\text { 19. Depresiones ciclónicas, } \\
\text { perturbaciones, huracanes. }\end{array}$ & 90 & 1554 & 1914 & 2036 \\
\hline
\end{tabular}

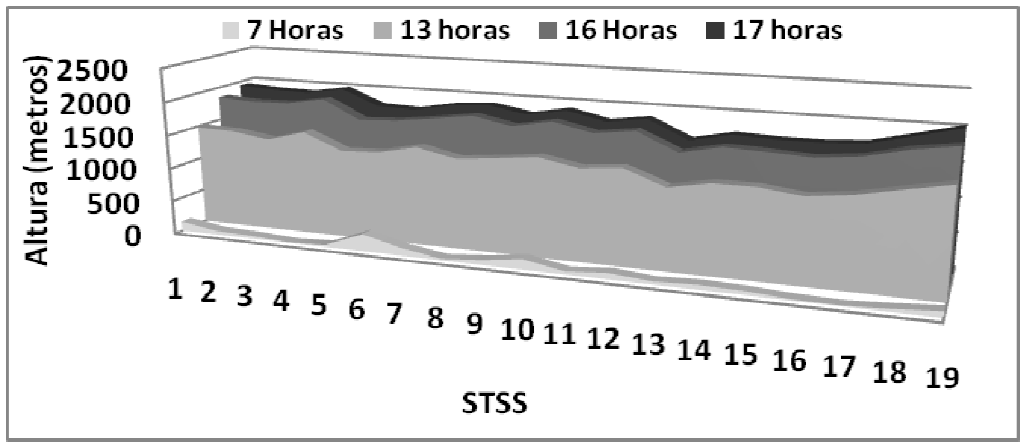

Figura 34 - Altura promedio de la capa de mezcla para cada TSS, en la ciudad de Pinar del Río en el período 2006-2010, a las 7, 13, 16 y 17 horas.

debido a una mayor presencia de la categoría muy inestable, mientras que en las horas nocturnas ocurre lo contrario producto de una mayor presencia de la categoría muy estable.

- En los meses de verano se experimenta mejores condiciones para la dispersión de los contaminantes atmosféricos en la variación mensual de la estabilidad atmosférica, debido a una mayor presencia de la categoría muy inestable en estos meses, producto de una transición más rápida desde la categoría muy estable. 
- La variación mensual de la estabilidad atmosférica por horarios, mostró que a las 17 horas existen las mejores condiciones para la dispersión de los contaminantes, debido a un mayor dominio de la categoría muy inestable, mientras que a las 7 horas la categoría muy estable, es la de mayor ocurrencia, existiendo condiciones desfavorables para la dispersión vertical de contaminantes atmosféricos.

- La relación de la estabilidad atmosférica con los procesos sinópticos, mostró que los valores de mayor ocurrencia se corresponden con el anticiclón del atlántico (TSS I) para los horarios 13, 16 y 17, siendo la categoría muy inestable la de mayor presencia, debido a la poca fuerza del viento y a las altas temperaturas que se experimentan en estos horarios. Mientras que a las 7 horas la categoría muy estable fue la de mayor ocurrencia, bajo el predominó de los anticiclones migratorios (TSS VIII), a esta hora la ausencia del sol en la mayoría de los meses del año es determinante para que la atmósfera tenga este comportamiento, unido a la disminución gradual de la fuerza del viento que experimenta el TSS VIII en su transito hacia el Océano Atlántico.

- Las mejores condiciones para la dispersión de los contaminantes atmosféricos, en la variación horaria de la altura promedio de la capa de mezcla atmosférica, se observa en las horas diurnas, alcanzando el máximo valor a las 17 horas, llegando muy próximo a los 1900 $\mathrm{m}$, mientras que en las horas nocturnas no se alcanzan los $200 \mathrm{~m}$ de altura.

- Las mejores condiciones para la dispersión de los contaminantes atmosféricos en la variación mensual de la altura promedio de la capa de mezcla atmosférica, se experimentó en los meses de verano, siendo máximo este comportamiento en mayo, entando muy próximo a los $750 \mathrm{~m}$ de altura, mientras que en los meses invernales estuvo alrededor de los $500 \mathrm{~m}$ de altura.

- La variación mensual de la altura promedio de la capa de mezcla atmosférica por horarios, mostró las mejores condiciones para la dispersión de los contaminantes en la vertical a las 17 horas, sobrepasando los $2000 \mathrm{~m}$ en los meses de abril y mayo, mientras que las peores condiciones existieron a las 7 horas y no alcanzó los $300 \mathrm{~m}$.

- La relación de la altura promedio de la capa de mezcla atmosférica con los procesos sinópticos, mostró que la mayor altura ocurrió a las 17 horas (2182 m), bajo la influencia de situaciones ciclónicas (TSS IV), generando así la mayor fuerza del viento, unido a la alta turbulencia atmosférica imperante por el aumento gradual de la temperatura ambiente a lo largo del día.

\section{REFERENCIAS BIBLIOGRÁFICAS}

ALONSO, A. Estudio de algunos factores meteorológicos que influyen en la dispersión de la contaminación atmosférica en La Habana. Trabajo de Diploma. INSTEC, p.22-55, 2013.

ÁLVAREZ, R. Estudio de la dispersión de contaminantes en la atmósfera de Cuba. Tesis de Doctor en Ciencias FísicoMatemáticas. IFA, Moscú, p.39-66, 1976.

BATCHVAROVA, E.; GRYNING, S. E. Applied model for the growth of the daytime mixed layer. Boundary Layer Meteorology, v. 56, p. 261-274, 1991.

DEARDORFF, J. W. Numerical investigation of neutral and unstable planetary boundary layers. Journal of the Atmospheric Sciences, v. 29, p. 91-115, 1972.

EPA. AERMOD. Description of model formulation. EPA454/R-03-004, Office of Air Quality Planning and Standards, p. 91, 2004a.

EPA. User's Guide for the Industrial Source Complex (ISC3) Dispersion Models - Volume I. EPA-454/B-95-003a, 1995.

FRIEDMAN, M. The use of ranks to avoid the assumption of normality implicit in the analysis of variance. Journal of the American Statistical Association, v. 61, p. 1081-1096, 1937.

GARRATT, J. R. The atmospheric boundary layer, Cambridge University Press, p. 316, 1992.

GILL, A. E. Atmospheric-Ocean Dynamics. Academic Press, p.54, 1982.

GONZÁleZ, Y. MLHASC Mixing Layer Heigth and Atmospheric Stability Condition. Instituto de Meteorología (INSMET), La Habana, Cuba, 2013.

GRYNING, S. E et al. On the extension of the wind pro_le over homogeneous terrain beyond the surface layer. BoundaryLayer Meteorology, v. 124, p. 251, 2007.

HAYES, S. R.; MOORE, G. E. Air quality model performance: a comparative analysis of 15 model evaluation studies. Atmospheric Environment, v.20, p.1897-1911, 1986.

IZUMI, Y. Kansas 1968 Field Program Data Report. No. 379, AFCRL-72-0041, Air Force Cambridge Research Laboratory, Bedford, MA, p. 79, 1971.

LAMB, R. G. Diffusion in the convective boundary layer. Atmospheric Turbulence and Air Pollution Modelling. Nieuwstadt, F. T. M. and van Dop, H., Reidel, p. 159-229, 1982.

LAPINEL, B. La circulación atmosférica y las características espacio temporales de las lluvias en Cuba. Tesis para la obtención del grado científico de candidato a Doctor en Ciencias Geográficas. p. 123, 1988.

LÓPEZ, C. M. Factores meteorológicos en la propagación de contaminantes del aire en la Ciudad de La Habana. 
Informe Científico-Técnico no. 46, INSMET, ACC, p. 78, 1978.

MONIN, A. S.; A. OBUKHOV, M. Basic laws of turbulent mixing in the ground layer of the atmosphere. Akad. Nauk. SSSR, Geofiz. Inst. Tr., v.151, p.163-187, 1954.

NIEUWSTADT, F. T. M.; VAN DOP, H. Atmospheric Turbulence and Air Pollution Modelling. Reidel, p. 358, 1982.

PASQUILL, F. The estimation of the dispersion of wind-borne material, Meteorological Magazine, v. 90, p. 33-49, 1961.

PASQUILL, F.; SMITH, F. R. Atmospheric Diffusion. John Wiley and Sons Inc., New York, p. 440, 1983.

READINGS, C. J. et al. The 1973 Minnesota tmospheric boundary layer experiment. Weather, v. 29, p. 309-312, 1974.

RODRÍGUEZ, D. et al. Estudio de variables meteorológicas secundarias que intervienen en la difusión de contaminantes atmosféricos. Caso de estudio ciudad de Pinar del Río. Revista Cubana de Meteorología, v. 18, n. 1, p.35-50, 2012a.

RODRÍGUEZ, D. et al. Estudio de variables meteorológicas secundarias que intervienen en la difusión de contaminantes atmosféricos. Caso de estudio la comunidad de Santa Lucía. Revista Cubana de Meteorología, v. 18, n. 2, p.77-92, $2012 b$.

RODRÍGUEZ, D. et al. Resultados preliminares de cálculo de la altura de la capa de mezcla a través del uso del Global Forescast System. Convención Trópico. ISBN 978-959-282-079-1, p. 72-89, 2008. La Habana, Centro de Convenciones Capitolio, Cuba, p. 16-24, 2008.

RODRÍGUEZ, D. Sistema Automatizado de Gestión de Información de Fuentes Contaminantes (SAGIFC). Publicación electrónica del IV Congreso Cubano de Meteorología. ISBN: 978-959-7167-12-9. La Habana, Centro de Convenciones Capitolio, Cuba, p. 7-18, 2007.
SMITH, M. E. Review of the attributes and performance of 10 rural diffusion models. Bulletin of the American Meteorological Society, v. 65, p. 554-558, 1984.

THOMSON, D. J. The meteorological input module, ADMS 3 Technical Specification. Cambridge Environmental Research Consultants, p. 116, 2000.

TURNER, D. B. A difusión model for an urbana rea. Journal of Applied Meteorology, v. 3, p. 83-91, 1964.

TURTÓS, L. et al. Method for the estimation of the convective mixing height aimed to atmospheric local dispersion modeling. Aprobado para publicar como capítulo del libro Environmental Impact Assessments, ISBN: 978-1-60692667-3, Nova Science Publishers, Inc, 2009.

TURTÓS, L. et al. Metodología de estimación de variables meteorológicas secundarias para modelos de dispersión de contaminantes atmosféricos, Contribución a la Educación y la Protección Ambiental, v. 4, p. 266-277, 2003.

VENKATRAM, A. Dispersion in the stable boundary layer. Lectures on Air Pollution Modeling. Journal of Climate, v. 1, p. 229-265, 1988.

VENKATRAM, A.; WYNGAARD, J. C. Lectures on Air Pollution Modeling. Journal of Climate, v.1, p.390, 1988.

WEIL, J. C. Dispersion in the convective boundary layer. Lectures on Air PollutionModeling. Journal of Climate, v. 1, p.167-227, 1988.

WEIL, J. C. Updating applied diffusion models. Journal of Applied Meteorology, v. 24, n. 11, p. 1111-1130, 1985.

WILCOXON, F. Individual compariosons by ranking methods. Biometrics, v. 1, p. 80-83, 1945. 